\title{
Binaries as precious tools for stellar evolution and asteroseismology
}

\author{
Carla Maceroni \\ INAF - IAPS, via del Fosso del Cavaliere 100, 00133, Rome, Italy
}

\begin{abstract}
Eclipsing double-lined binaries had a fundamental role in laying the foundations of stellar astrophysics and are still today an invaluable tool, as they provide an independent, purely geometrical, and accurate determination of the stellar masses and radii (often with uncertainties lower than $1 \%$ ). This paper briefly reviews some important contributions to the field of stellar structure and to the more recent one of asteroseismology.
\end{abstract}

Keywords: binaries: eclipsing - binaries: spectroscopic - stars: evolution - stars: interiors - stars: pulsation

\section{Introduction}

Together with clusters binaries are the best testing tool for stellar models. As cluster members, binary components can be assumed to have the same age and chemical composition. Moreover, if the binary is eclipsing and both spectra are detected (EB-SB2), the fundamental parameters (masses, radii) can be derived without any assumption on their radiative properties. The accuracy of their determination, routinely around $1 \%$ but in the best cases lower than $0.5 \%$, provides stringent tests for theoretical models.

An enlightening example of the importance of binaries is the role played by a single system, YY Gem, in the knowledge of low mass stars.

YY Gem is a component of a multiple system (Castor $=\alpha \mathrm{Gem})$. This mini-cluster contains six stars in three binary systems: Castor A and B are single-lined spectroscopic binaries (SB1), while Castor C (YY Gem) is an EB-SB2. YY Gem is formed by two almost twin low-mass M stars (with $M \simeq 0.6 M_{\odot}, R=0.62 R_{\odot}, T_{\text {eff }} \simeq 3800 \mathrm{~K}$ ) in a close orbit (orbital period $P=0.814^{\mathrm{d}}$ ). Together with Kruger 60A, a visual binary with M2/M3 components, Castor C has been since the 1920's one of the few known binary with late type components, and that with the highest accuracy of masses and radii. These were derived from the Van Gent $(1926,1931)$ light curve and the Joy \& Sandford (1926)\& Struve, Herbig \& Horak (1950) radial velocities with an accuracy around 5\%. The component effective temperatures were estimated to be $3600 \mathrm{~K}$, i.e. slightly lower (by $200 \mathrm{~K}$ ) than more recent determinations. For this reason the system has always been the primary test for theoretical models of low mass stars.

In the 1950s stellar models had developed at the point of including energy generation by nuclear reactions, they however still badly failed in reproducing the properties of red dwarfs. The available models, which were based on p-p chain energy generation and radiative envelopes, gave for low mass stars a large luminosity excess. It was, indeed, the comparison with red dwarfs in binaries which 
motivated Osterbrock (1953) to compute the first models with convective envelopes. In his pivotal paper he showed that the properties of YY Gem could be better fitted by stellar structures including a convective zone with depth of the order of $\sim 30 \%$ the stellar radius, and that the alternative hypothesis of peculiar chemical abundance could be discarded. Besides, he pointed out that his new convective models did not work for the later system, $\mathrm{Kr} 60 \mathrm{~A}$, for which, later on, a fully convective structure was postulated by Limber (1958a,b).

As we will see in the next section, however, the discrepancies between models and observations for red dwarfs were only temporarily solved by the model improvement, as the increasing accuracy of parameter determination and a larger and larger sample of low mass binary components evidenced smaller but persisting deviations.

Binaries have also been largely used to test the need, and the behaviour with mass, of core overshooting in small and intermediate mass stars. The question has longly been debated during the last decades and a definitive conclusion has not yet been reached (Section 3).

Another recent new field in which binaries are being used as an effective test tool is asteroseismology of red giants. The masses and radii derived from asteroseismology thanks to the "scaling relations", which require however an estimate of the effective temperature, can be compared with the independent determination of oscillating giants in binary systems (Section 4). The validation of such relations is extremely important also for the study of the galactic structure and history.

\section{Binaries and the mass-radius relation for red dwarfs}

Figure 1 shows the masses and radii of binaries known with an accuracy of $2 \%$. The data are from the DEBcat catalog (Southworth 2005), for a cumulative comparison with theoretical models an isochrone is also shown (300 My, from Baraffe et al. 1998). This isochrone is illustrative of the zero age main sequence for low mass stars and two of the binary systems depicted in Figure 1 (CU Cnc and YY Gem) are indeed in this age range. As it is well known, most red dwarf radii from binaries are larger than those expected from models. The face value of the deviation amounts to $\sim 10-15$ $\%$, which can be reduced to 5-10\%, but not cancelled, taking into account the star-to-star age and metallicity variation (Feiden \&Chaboyer 2012a). In particular, YY Gem radii are $10 \%$ larger than computed (Torres\& Ribas 2002).

The cause of the disagreement was searched in the missing ingredients of stellar models. It was soon noted that most known low-mass binary components are active stars, as they are in short period system and rotate fast due to spin-orbit synchronisation. It was, therefore, suggested that the binary components could be inflated by the effect of strong magnetic fields (Mullan \& Mac Donald 2001). Magnetic activity was evident from the light curves, with out-of eclipse variations due to spots, and from other indicators. In particular López-Morales (2007), studying a sample of EBs and single stars with interferometric radius determination, found a clear correlation between radius deviation and coronal X-ray emission.

Different hypothesis on the role of magnetic field have been formulated, invoking strong global magnetic fields which were supposed to inhibit convection, or local strong concentration of magnetic fields and dark starspot on the surface. The latter ones can produce larger and cooler stars by trapping energy at their base (Spruit 1982). Suppression of convection can be produced by magnetic fields of reasonable intensity in non fully convective red dwarfs (Mullan \& Mc Donald 2001, Feiden \& Chaboyer 2012b), However, fully convective red dwarfs component (such as in the other well studied system CM Dra) require MGauss fields, and local field concentration seems to be a more viable solution. While there are still details to be explained, such as a few long period binaries with slow rotating components and nevertheless inflated radii (Irwin et al. 2011, Winn et al. 2011), there is a 


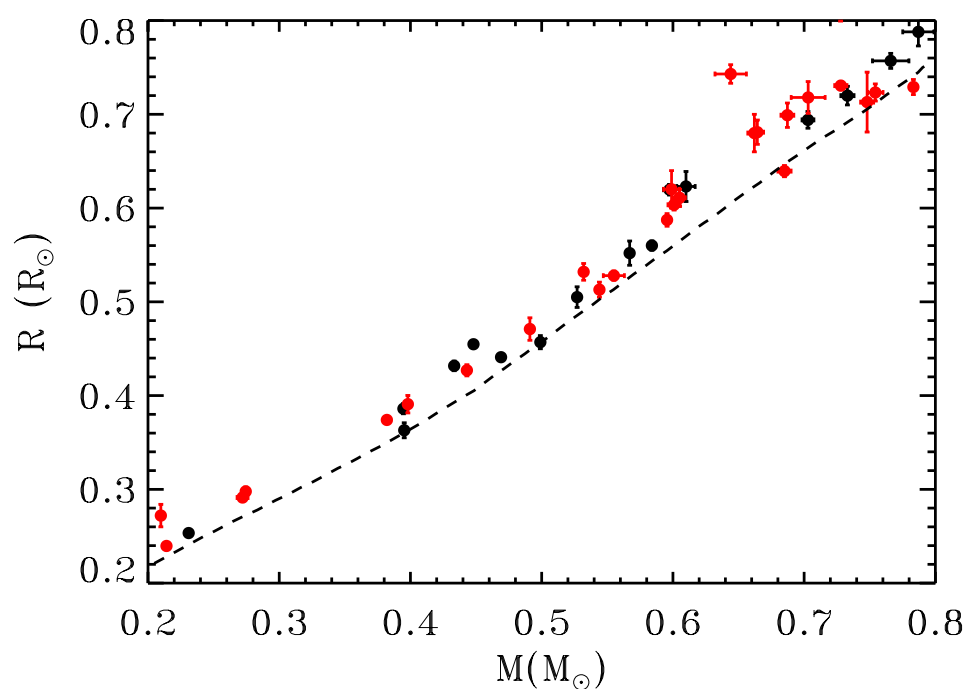

Figure 1: Mass vs. radius for the low mass components of binary systems, as collected in the DEBcat current version (Southworth 2015), black dots denote primaries (i.e. the more massive component in a pair), red dots secondaries.

general consensus about the magnetic origin of the phenomenon. An excellent review of the topic, exploring as well hypotheses different from magnetic fields, can be found in Felden (2015).

\section{Binaries and core overshooting}

A still persisting weakness of stellar models is the treatment of mixing at the convective boundary of stellar cores. The Schwartzschild criterion, defining stability against convection, implies zero acceleration of the convective elements which can, however, have a non-zero velocity and penetrate the upper stable layers (the so called overshooting). This extra-mixing increases the amount of available nuclear fuel and changes the Main Sequence (MS) lifetime of stellar models as well as the speed of evolution and the luminosity of post-MS stars. The first studies on the subject date back to the 1960s (Roxburgh 1965, Saslaw \& Schwarzschild 1965) and a long series of papers dealt with the need of extra-mixing and its possible dependence on the stellar mass. We only recall here a few recent ones: the series of papers by Claret \& Torres $(2016,2017,2018)$ comparing the stellar models to EB-SB2, the similar recent work by Constantino \& Baraffe (2018), and the statistical study on the uncertainties of calibration by binaries by Valle et al. (2016). An exhaustive list of others works can be found in the above-mentioned papers.

The classical description of overshooting is in terms of the parameter $\alpha_{\mathrm{ov}}=d_{\mathrm{ov}} / H_{\mathrm{p}}$, where $d_{\mathrm{ov}}$ is the depth of penetration beyond the Schwarzschild core and $H_{\mathrm{p}}$ is the pressure scale height. That implies an additional fully mixed region of depth $d_{\mathrm{ov}}$. A more recent popular description supposes, instead, that convective cells gradually disintegrate by a diffusive process. In this case a different parameter describes the process: $f_{\mathrm{ov}}=H_{\nu} / H_{\mathrm{p}}$, where $H_{\nu}$ is the velocity scale height (Herwig et al. 1997). An approximate relationship between $\alpha_{\mathrm{ov}}$ and $f_{\mathrm{ov}}$ has been proposed by Claret \& Torres (2017) and allows to compare the results obtained with the two different descriptions: $\alpha_{\mathrm{ov}} / f_{\mathrm{ov}}=$ $11.36 \pm 0.22$.

Stellar models with overshooting have been tested against stellar clusters and, of course, the best known binaries. Claret \& Torres, in the above-mentioned series of papers, selected a sample of about thirty double-lined eclipsing binaries with masses in the range $1.2-4.4 M_{\odot}$, parameters with 
accuracy of $3 \%$, and with several components evolved enough to show the effects of overshooting. Their detailed study confirmed previous suggestions: the fitting of binaries requires overshooting in most cases and shows a clear mass dependence. The derived value of $f_{\text {ov }}$ increases sharply between $1.2-2.0 M_{\odot}$ and remains then constant for the larger masses (see Fig. 2 of Claret \& Torres 2018). This behaviour does not depend on the chemical element mixture nor on the description of overshooting. Besides, even the change of evolutionary code (from Granada one in the 2016 paper to MESA in following ones) did not affect the result.

In contrast, however, to these conclusions the recent study by Constantino \& Baraffe (2018) depicts a much less defined scenario, at least for lower mass stars, those below $2 M_{\odot}$. These authors used a subsample of the Claret and Torres binaries, selecting it to be representative of the whole sample, and a similar fitting procedure but a different evolutionary code (MONSTAR, Campbell \& Lattanzio 2008). While their best fitting models for the binary components are quite similar to those by Claret and Torres, other acceptable solutions with different overshooting values could also be found, at least for the masses below $2.0 M_{\odot}$. The consequence is a large range of acceptable values of $f_{\text {ov }}$ below 2.0 $M_{\odot}$, which completely hides the mass trend. In conclusion they could not constrain overshooting in binaries with Main Sequence or subgiant components, but only for more evolved stars.

The same difficulty is confirmed by a different approach, based on a statistical tests on models rather than comparison with observed systems, Valle et al $(2016,2018)$. These authors try to recover the parameters (including overshooting) of synthetic binaries by means of a grid-based maximum likehood pipeline, which employs the same grid of models used to build the mock stars. The computed parameters of the synthetic binaries were assumed to be affected by the typical observational errors of real systems and the inverse procedure was applied to derive the core sizes of synthetic systems with MS components in the mass range $1.1-1.6 M_{\odot}$. Their conclusion is that the calibration of the convective core overshooting with double-lined eclipsing pairs on the Main Sequence is poorly reliable, being the uncertainties so large to make impossible to constrain the overshooting parameter. On the other hand they also confirmed that the scenario changes for more evolved components (and that overshooting is then a necessary ingredient of models).

Other observables are needed to add further constraints to the models. There is good hope, for instance, that asteroseismic analysis could help. Recently Deheuvels et al. (2016) tried to measure the convective core size in a sample of MS Kepler targets with mass around the limit for the presence of a convective core $\left(1.1 M_{\odot}\right.$ at solar metallicity). Using a seismic diagnostic based on the small and large separation ratios for radial and dipolar modes, they could estimate the core size of eight MS stars. These resulted to be larger than the Schwarzschild limit suggesting the presence of overshooting. It has to be noticed that for solar type pulsators an estimate of the stellar mass can be obtained by asteroseismic analysis, though with an accuracy still far from that of binaries. In the future however we can expect improvements of the available data, thanks to future missions as PLATO, and a parallel development of the theoretical tools.

\section{Binaries and the scaling relations for oscillating giants}

In the last years binaries with accurate parameters (mainly EB-SB2) containing oscillating red giant components have been important test object to validate the asteroseismic "scaling relations", which hold for the solar-like pulsators. These relations connect some easy-to-determine global properties of oscillations to the mass and radius of the pulsator. The oscillation spectrum of solar-like pulsators (as G-K red giants) can be described by two global parameters, the frequency at the maximum power, $\nu_{\max }$, and the mean frequency separation between consecutive radial modes, $\Delta \nu$. These quantities, combined with an estimate of $T_{\text {eff }}$, allow to estimate the star surface gravity and mean density with respect to the sun (Ulrich 86; Brown et al.1991; Kjeldsen \& Bedding 1995; Belkacem et al. 2011), 


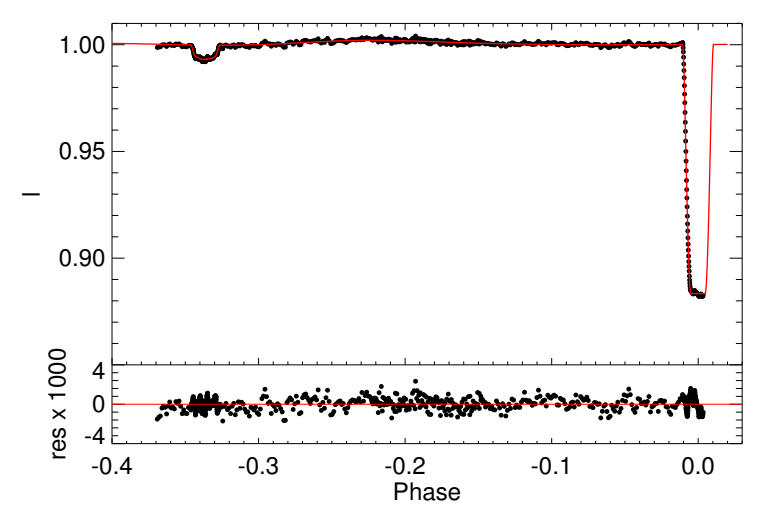

Figure 2: CoRoT light curve fit and corresponding residuals. The solution was performed for a detrended binned curve, while the residuals for the harmonic analysis were computed with respect to all the points.

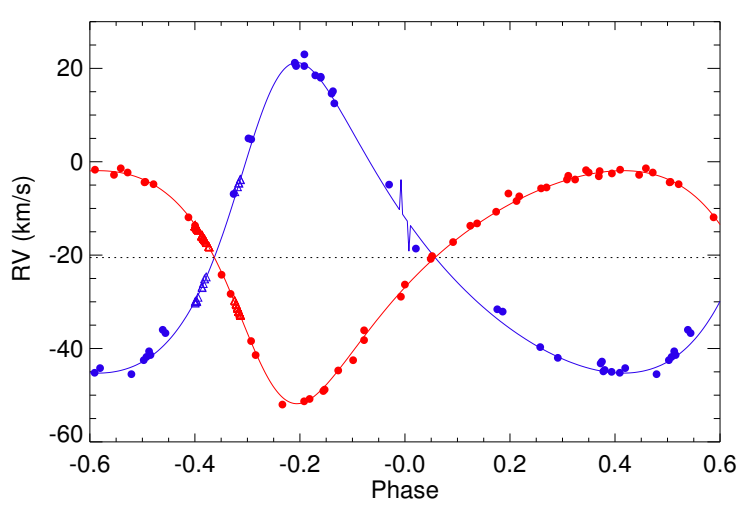

Figure 3: The radial velocity curves by Griffin \& Griffin (1986) (circles) and HARPS spectra (open triangles). A zero point correction of $-1.2 \mathrm{Kms}^{-1}$ was applied to the first data set.

according to :

$$
\frac{g}{g_{\odot}}=f_{\nu_{\max }} \frac{\nu_{\max }}{\nu_{\max , \odot}}\left(\frac{T_{\text {eff }}}{T_{\text {eff }, \odot}}\right)^{1 / 2} ; \quad \frac{\rho}{\rho_{\odot}}=f_{\Delta \nu}\left(\frac{\Delta \nu}{\Delta \nu_{\odot}}\right)^{2}
$$

where $f_{\nu_{\max }}$ and $f_{\Delta \nu}$ are correction factors to be estimated/calibrated for stars different from the Sun. The original formulation by Kjeldsen \& Bedding (1995) did not contain the correction factors, but the first tests in the case of giant components found in Kepler binaries (e.g. Gaulme et al. 2016) suggested some form of correction.

The poor current knowledge of excitation and damping of solar-like oscillations does not yet allow a theoretical estimate of $f_{\nu_{\max }}$, but empirical tests such as the comparison between the seismic and spectroscopic log g (e.g. Morel et al. 2014), or values from binary systems (Brogaard et al. 2015; Gaulme et al. 2016), have shown that $f_{\nu_{\max }} \simeq 1$. On the contrary, $f_{\Delta \nu}$ can be estimated from theoretical calculations, and turns out to depend on the stellar mass, effective temperature, evolutionary state, chemical composition, and even on the method used to estimate the reference solar value (Rodrigues et al. 2017, Huber et al 2017, and references therein).

The scaling relations for $\mathrm{M}$ and $\mathrm{R}$ can be readily be derived from Eqs 1:

$$
\frac{R}{R_{\odot}}=\frac{f_{\nu_{\max }}}{f_{\Delta \nu}} \frac{\nu_{\max }}{\nu_{\max , \odot}}\left(\frac{\Delta \nu}{\Delta \nu_{\odot}}\right)^{-2}\left(\frac{T_{\mathrm{eff}}}{T_{\mathrm{eff}, \odot}}\right)^{1 / 2} ; \quad \frac{M}{M_{\odot}}=f_{\Delta \nu}\left(\frac{\Delta \nu}{\Delta \nu_{\odot}}\right)^{2}\left(\frac{R}{R_{\odot}}\right)^{3}
$$

The relations have already been widely used in the study of galactic populations by means of pulsating giants (the so-called ensemble asteroseismology, eg. Miglio et al. 2013). However, their accuracy and validity-domain are still matter of debate (Huber et al.2012; Baines et al. 2014; Gaulme et al. 2016). Independent determination of mass and radius for giants indicate that the relations have an accuracy of 5-11\% for the asteroseismic radius (Miglio 2012; Huber et al 2017; Gaulme et al. 2016) and 1025\% for the mass (White et al. 2011; Miglio et al. 2013; Brogaard et al. 2016; Gaulme et al. 2016). The larger deviations are found for the plain version of the relations (i.e. when $f_{\nu_{\max }}=f_{\Delta \nu}=1$ ).

Furthermore it has to be noticed, for what concerns the mass range of validity, that most targets studied until now are stars of low-mass $\left(<2 M_{\odot}\right)$ and relatively small radius $\left(0.8-15 R_{\odot}\right)$.

For this reason, it was particularly interesting to detect solar-like oscillations in a giant component of a known system, which was observed as primary asteroseismic target in two runs of the CoRoT mission. The system, HR 6902 (= HD 169689), is a long period binary belonging to the $\zeta$ Aur class. 
Table 1: Orbital and physical parameters of HR 6902.

\begin{tabular}{|l|ccc|}
\hline & \multicolumn{3}{|c|}{ System } \\
& Primary & Secondary \\
\hline$i\left(^{\circ}\right)$ & \multicolumn{3}{|c|}{$86.223 \pm 0.007$} \\
$e$ & \multicolumn{3}{|c}{$0.315 \pm 0.002$} \\
$\omega\left(^{\circ}\right)$ & \multicolumn{3}{|c}{$144.02 \pm 0.16$} \\
$q$ & \multicolumn{3}{|c}{$0.750 \pm 0.007$} \\
$a\left(R_{\odot}\right)$ & \multicolumn{3}{|c}{$421 \pm 3.5$} \\
$\gamma\left(\mathrm{Kms}^{-1}\right)$ & \multicolumn{3}{|c}{$-20.50 \pm 0.04$} \\
$T_{\text {eff }}(\mathrm{K})$ & $4804^{a} \pm 70$ & $11073 \pm 500$ \\
$M\left(M_{\odot}\right)$ & $3.87 \pm 0.13$ & $2.90 \pm 0.14$ \\
$R\left(R_{\odot}\right)$ & $35.7 \pm 0.5$ & $2.97 \pm 0.04$ \\
$\log g$ & $1.92 \pm 0.02$ & $3.96 \pm 0.02$ \\
\hline
\end{tabular}

${ }^{\text {a }}$ Fixed value

That denotes binaries formed of a late-type giant and a hot dwarf, and are characterised by a composite spectrum, showing the superimposed features of both components. These systems, and especially the eclipsing pairs (EB-SB2), are excellent benchmarks of stellar evolutionary models (e.g. Claret 2009 and references therein). HR 6902, with a primary giant component of $\sim 4 M_{\odot}$, can also test the validity of scaling relations in an unexplored mass range. It is a suitable testing tool also because of its long period $\left(\simeq 385^{\mathrm{d}}\right)$. That is on one side a disadvantage for data collection, as a long time span is needed to get the light and radial velocity curves, but on the other makes sure that duplicity does not play a role, through tidal action: tidal forces strongly depend on distance and in HR 6902 the giant radius is only $8 \%$ of the system semiaxis.

HR 6902 was observed in the CoRoT asteroseismic field in two long runs: LRc04 and LRc10. Each run lasted $\sim 90$ continuous days and provided $\sim 200000$ points with sampling of $32^{\mathrm{s}}$ and a very high accuracy $(0.03 \%)$. A detrended and binned version of the light curve is shown in Fig.2. The radial velocity curves displayed in Fig. 3 were obtained from extant and unpublished observations by E.R. Griffin and collaborators, and thanks to an ESO large program with the HARPS spectrograph (Poretti et al. 2013).

The complete analysis of this system will be published elsewhere (Maceroni et al. 2019, Maceroni et al. in preparation). In short, we performed preliminary non-simultaneous light and radial velocity curve fits by differential correction with PHOEBE (Prša \& Zwitter 2005). The global minimum of the cost function (in our case the sum of the squared residuals) was then found with FITBINARY (Maceroni et al. 2014), a wrapper connecting the genetic algorithm PIKAIA (Charbonneau 1995) to the PHOEBE binary modelling code. Different FITBINARY runs were performed for the LC and the RV data. The final synthetic light curve, corresponding to the best system model, was subtracted from the data to obtain the residuals for the harmonic analysis.

The residuals of the light curve solution were then analysed to detect pulsations. The power spectrum of Fig.4 was fitted with two background components and a Gaussian excess power law. The "universal red giant oscillation pattern" (Mosser et al. 2011) was used to derive $\Delta \nu$. That yielded:

$$
\nu_{\max }=10.2 \pm 0.4 \mu \mathrm{Hz} \quad \Delta \nu=1.17 \pm 0.01 \mu \mathrm{Hz}
$$

These corresponding asteroseismic values of $\log g$ and $\rho$ are: $\log g=1.92 \pm 0.09$ and $\rho=$ $(7.5 \pm 0.2) \cdot 10^{-5} \rho_{\odot}$. The comparison with Table 1 confirms and extends the results of Gaulme et al. (2016) to a high mass giant in a wide binary, namely: the gravity is in excellent agreement with the 


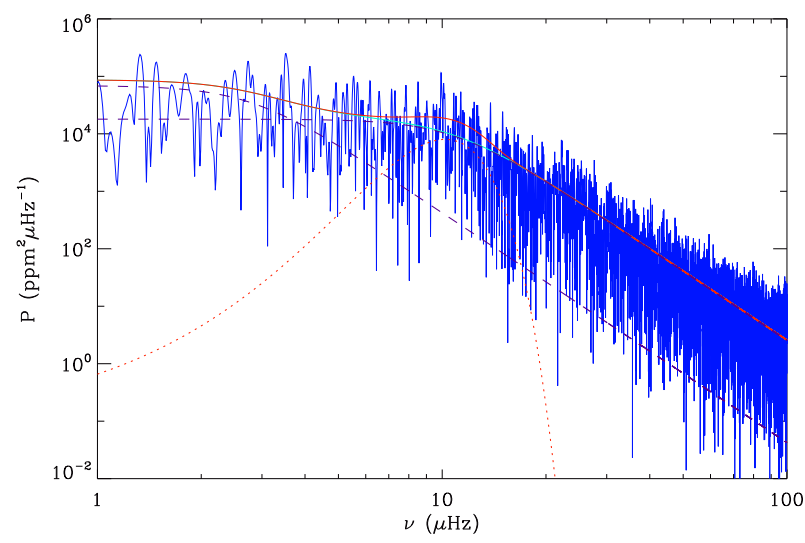

Figure 4: Power spectrum of HR 6902 fitted with two background components (dashed lines) and a Gaussian excess power centered on $\nu_{\max }($ dotted line).

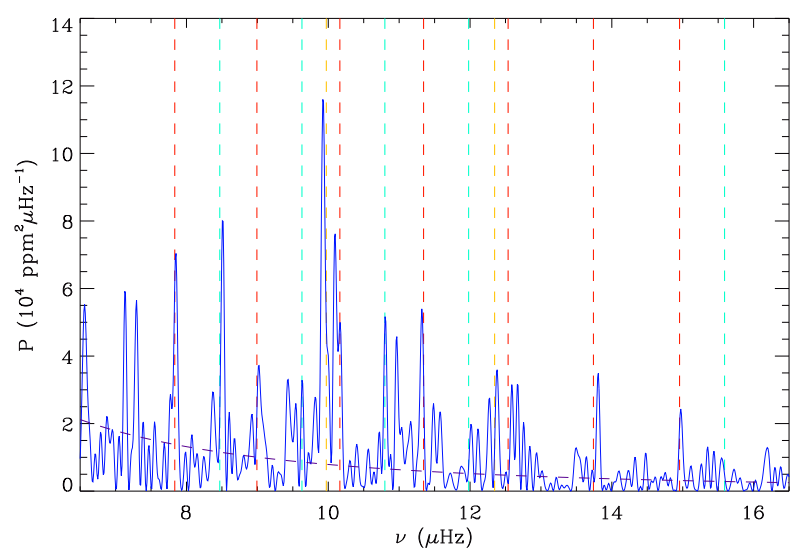

Figure 5: Identification of the low-degree oscillation spectrum (radial modes in red, dipole modes in light blue, quadrupole modes in orange).

dynamical value, while density results to be smaller by $10 \%$. To bring into agreement the seismic and dynamical values a correction $f_{\Delta \nu}=1.3$ is needed.

\section{Conclusions}

In this paper we have presented some topics in stellar physics which have largely benefited from the use of binaries to test the interior structure and evolution of stars. The dynamical masses and stellar radii obtained from EB-SB2 are still the best testbed for stellar models, at least when the influence of duplicity on the derived parameters is excluded (as in the case of wide, non-interacting systems).

Space missions, such as CoRoT and Kepler, have provided photometry of unprecedented accuracy and timespan. Often the bottle neck is the acquisition of spectroscopic measurements (radial velocity curves) which require the availability of large telescopes over long time intervals. Dedicated long campaign are necessary and have to be planned as mission follow-up.

In the future the sinergy between asteroseismology and binary studies and the availability of PLATO data will hopefully help to solve some of the still standing problems, such as the longly debated question of the dependence of overshoting on stellar mass.

\section{Acknowledgements}

I gratefully thank the Symposium organisers for their kind invitation and the INAF - IAPS for hospitality and travel support. Special thanks to Josefina Montalbán for useful discussions and advice and to R.E. Griffin for providing her unpublished data of HR 6902. Finally, I heartily thank Arlette Noels for the fruitful scientific interaction and the pleasant time spent together during the years of the CoRoT mission.

\section{References}

Baines K., Ellyn K., Armstrong J. T. et a. 2014, ApJ 781, 90

Baraffe I., Chabrier G., Allard F., Hauschildt P. H. 1998, A\&A, 337, 403 
Belkacem K. 2011, LNP, 832, 139

Brogaard K., Jessen-Hansen J., Handberg R. et al. 2016, AN 337, 793

Brown, T.M., Gilliland R.L., Noyes, R.W. et al. 1991, ApJ, 368, 599

Campbell,S. W., Lattanzio J. C. 2008, A\&A, 490, 769

Charbonneau P. 1995, ApJS 101, 309

Claret A. 2009, A\&A 507, 377

Claret A., Torres G. 2016, A\&A, 592, A15

Claret A., Torres G. 2017, ApJ, 849, 18

Claret A., Torres G. 2018, ApJ, 859, 100

Constantino T., Baraffe I. 2018, A\&A, 618, A177

Deheuvels S., Brandão I., Silva Aguirre V. et al. 2016, A\&A 589, 93

Feiden G. A., Chaboyer B. 2012a, ApJ, 757, 42

Feiden G. A., Chaboyer B. 2012b, ApJ, 761, 30

Felden G. 2015, ASPC, 496, 137

Gaulme P., McKeever J., Jackiewicz J. et al. 2016, ApJ, 832,121

Griffin R. and R. 1986, J. Astrophys Astr. 7, 195

Herwig F., Bloecker T., Schoenberner D. et al. 1997, A\&A, 384, L81

Huber D., Ireland M. J, Bedding T. R. et al. 2012, ApJ 760,32

Huber D, Zinn, J., Bojsen-Hansen M. et al. 2017, ApJ, 844, 102

Irwin J. M., Quinn S. N., Berta Z. K. et al. 2011, ApJ, 742, 123

Joy A. H., Sanford R. F. 1926, ApJ, 64, 250

Kjeldsen H., Bedding T. 1995, A\&A, 293,87

Limber D. N. 1958a, ApJ, 127, 363

Limber D. N. 1958b, ApJ, 127, 387

López-Morales M. 2007, ApJ, 660, 732

Maceroni C., Lehmann H., da Silva, R. et al. 2014, A\&A 563, A59

Maceroni C, Montalbán J., Da Silva R. et al. 2019, IAUS 339, Griffin E.R. Ed, CUP

Miglio A. 2012, ASPP 26,11

Miglio A. Chiappini C., Morel T. et al. 2013, MNRAS 429, 433

Morel T., Miglio A., Lagarde N. et al. 2014, A\&A 564, 119

Mosser B., Belkacem K., Goupil M. J. et al. 2011, A\&A 525, L9

Mullan D.J., MacDonald J. 2001, ApJ 559, 353

Osterbrock D. E. 1953, ApJ, 118, 529

Poretti E., Rainer M., Mantegazza L. et al. 2013, ASSP, 31, 39

Prša A., Zwitter T. 2005, ApJ, 628, 426

Rodrigues T., Bossini D., Miglio A. et al. 2017, MNRAS 467, 1433

Roxburgh I. W 1965, MNRAS, 130, 223

Saslaw W. C. ,Schwarzschild M., 1965, ApJ 142, 1468

Southworth J. 2005, ASPC, 496, 164

Spruit H. C. 1982, A\&A, 108, 348

Struve O., Herbig G., Horak H. 1950, ApJ, 112, 216

Torres G., Ribas I. 2002, ApJ, 567, 1140

Ulrich R.K. 1986, ApJ 306, L37

Valle G., Dell'Omodarme M., Prada Moroni P. G. et al. 2016, A\&A, 587, A16

Valle G., Dell'Omodarme M., Prada Moroni P. G. et al. 2018, A\&A, 615, A62

van Gent H. 1926, BAN, 3, 121

van Gent H. 1931, BAN, 215, 99

White T.R., Bedding T. R.; Stello D. et al. 2011, ApJ 743, 161

Winn J. N., Albrecht S., Johnson J. A., et al. 2011, ApJL, 741, L1 